\title{
Effectiveness and safety of reduced dose non-vitamin K antagonist oral anticoagulants and warfarin in patients with atrial fibrillation: propensity weighted nationwide cohort study
}

\author{
Peter Brønnum Nielsen, ${ }^{1}$ Flemming Skjøth, 1,2 Mette Søgaard, 1,3 Jette Nordstrøm Kjældgaard, 1,3 \\ Gregory Y H Lip, ${ }^{1,4}$ Torben Bjerregaard Larsen ${ }^{1,3}$
}

${ }^{1}$ Aalborg Thrombosis Research

Unit, Department of Clinical

Medicine, Faculty of Health,

Aalborg University, Aalborg,

Denmark

2Unit of Clinical Biostatistics, Aalborg University Hospital, Denmark

${ }^{3}$ Department of Cardiology, Aalborg University Hospital, DK-9000, Denmark

4University of Birmingham, Institute of Cardiovascular Sciences, City Hospital,

Birmingham, UK

Correspondence to: T B Larsen tobl@rn.dk

Cite this as: $B M J$ 2017;356:j510 http://dx.doi.org/10.1136/bmj.j510

Accepted: 08 January 2017

\section{ABSTRACT}

OBJECTIVE

To examine clinical effectiveness and safety of apixaban $2.5 \mathrm{mg}$, dabigatran $110 \mathrm{mg}$, and rivaroxaban $15 \mathrm{mg}$ compared with warfarin among patients with atrial fibrillation who had not previously taken an oral anticoagulant.

DESIGN

Propensity weighted (inverse probability of treatment weighted) nationwide cohort study.

SETTING

Individual linked data from three nationwide registries in Denmark.

\section{PARTICIPANTS}

Patients with non-valvular atrial fibrillation filling a first prescription for an oral anticoagulant from August 2011 to February 2016. Patients who filled a prescription for a standard dose non-vitamin $\mathrm{K}$ antagonist oral anticoagulant (novel oral anticoagulants, NOACs) were excluded. To control for baseline differences in the population, a propensity score for receipt of either of the four treatment alternatives was calculated to apply an inverse probability treatment weight.

\section{INTERVENTION}

Initiated anticoagulant treatment (dabigatran $110 \mathrm{mg}$, rivaroxaban $15 \mathrm{mg}$, apixaban $2.5 \mathrm{mg}$, and warfarin).

\section{MAIN OUTCOME MEASURES}

Patients were followed in the registries from onset of treatment for the primary effectiveness outcome of ischaemic stroke/systemic embolism and for the principal safety outcome of any bleeding events.

RESULTS

Among 55644 patients with atrial fibrillation who met inclusion criteria, the cohort was distributed according

\section{WHAT IS ALREADY KNOWN ON THIS TOPIC}

Use of reduced dose non-vitamin K antagonist oral anticoagulants (NOAC) has been increasing since their introduction

Limited evidence exists relating to effectiveness and safety of reduced doses compared with warfarin based on data from clinical practice

\section{WHAT THIS STUDY ADDS}

Rates of ischaemic stroke or systemic embolism with apixaban $2.5 \mathrm{mg}$ were higher but not significantly so compared with warfarin

Dabigatran $110 \mathrm{mg}$ and rivaroxaban $15 \mathrm{mg}$ both showed a trend towards lower thromboembolic rates, but rates were not significantly different to rates with warfarin

Rates of bleeding, the principal safety outcome, were not significantly different for apixaban and rivaroxaban compared with warfarin but were lower for dabigatran to treatment: apixaban $n=4400$; dabigatran $n=8875$; rivaroxaban $n=3476$; warfarin $n=38893$. The overall mean age was 73.9 (SD 12.7), ranging from a mean of 71.0 (warfarin) to 83.9 (apixaban). During one year of follow-up, apixaban was associated with higher (weighted) event rate of ischaemic stroke/systemic embolism (4.8\%), while dabigatran, rivaroxaban, and warfarin had event rates of $3.3 \%, 3.5 \%$, and $3.7 \%$, respectively. In the comparison between a non-vitamin $\mathrm{K}$ antagonist oral anticoagulant and warfarin in the inverse probability of treatment weighted analyses and investigation of the effectiveness outcome, the hazard ratios were 1.19 ( $95 \%$ confidence interval 0.95 to 1.49 ) for apixaban, 0.89 (0.77 to 1.03) for dabigatran, and 0.89 ( 0.69 to 1.16$)$ for rivaroxaban. For the principal safety outcome versus warfarin, the hazard ratios were 0.96 (0.73 to 1.27) for apixaban, 0.80 (0.70 to 0.92$)$ for dabigatran, and 1.06 (0.87 to 1.29) for rivaroxaban.

\section{CONCLUSION}

In this propensity weighted nationwide study of reduced dose non-vitamin $\mathrm{K}$ antagonist oral anticoagulant regimens, apixaban $2.5 \mathrm{mg}$ twice a day was associated with a trend towards higher rates of ischaemic stroke/systemic embolism compared with warfarin, while rivaroxaban $15 \mathrm{mg}$ once a day and dabigatran $110 \mathrm{mg}$ twice a day showed a trend towards lower thromboembolic rates. The results were not significantly different. Rates of bleeding (the principal safety outcome) were significantly lower for dabigatran, but not significantly different for apixaban and rivaroxaban compared with warfarin.

\section{Introduction}

Atrial fibrillation is associated with an increased risk of ischaemic stroke and mortality. During the past five years, large randomised trials have established that non-vitamin $\mathrm{K}$ antagonist oral anticoagulants (novel oral anticoagulants, NOACs) have a similar efficacy to warfarin but have a superior safety profile, largely driven by a substantial reduction in associated risk of intracranial haemorrhage. ${ }^{1}$ These findings have subsequently changed guidelines to either include NOACs as an option for stroke prophylaxis ${ }^{2}$ or to recommend these agents as first choice of drug. ${ }^{3}$ The findings of clinical trials have been confirmed in various observational cohort studies based on data from clinical practice. ${ }^{4-7}$

While meticulous dose adjustments are not required for NOACs as they are for warfarin, a clinical evaluation of appropriate (constant) dose is still necessary. Various degrees of renal function entails recommendations to offer these drugs in a reduced dosing regimen, but 
different cut-off values for age, body weight, or interacting drugs also require consideration for appropriate dose selection. ${ }^{8}$ Indeed, both age and chronic kidney disease in patients with atrial fibrillation intensifies the risk of stroke and increases the risk of bleeding during antithrombotic treatment. ${ }^{9}$ Elderly patients with atrial fibrillation (such as those aged $\geq 80$ ) and patients with impaired renal function were included in the landmark trials of NOACs, but these important subgroups comprised only a small proportion of the patient populations.

Nevertheless, contemporary guidelines suggest a reduced dosing regimen for dabigatran (110 mg twice a day) if patients are aged $\geq 80$ or have an estimated glomerular filtration rate (eGFR) of 30-50 mL/min; rivaroxaban (15 mg once a day) if eGRF is $15-49 \mathrm{~mL} / \mathrm{min}$; apixaban (2.5 mg twice a day) if two of the three following criteria are present: age $\geq 80$ or an eGRF $15-29 \mathrm{~mL} /$ min or body weight $\leq 60 \mathrm{~kg}$; or edoxaban (30 mg once a day) if eGFR is $15-50 \mathrm{~mL} / \mathrm{min} .{ }^{10}$ While subgroup analyses including patients with varying degree of renal function showed comparable efficacy with warfarin, ${ }^{11}$ evidence on reduce dose regimens of non-vitamin $\mathrm{K}$ antagonist oral anticoagulants from "real world" clinical practice is scarce. In response to this lack of evidence about reduce dose regimens for stroke prevention in atrial fibrillation, we conducted a nationwide cohort study to examine effectiveness and safety compared with warfarin. To ascertain appropriateness of prescribing, we particularly focused on subgroups of elderly patients and those with impaired renal function, given their potential "indication for dose reduction" when NOACs are used.

\section{Methods}

This was an observational cohort study of a nationwide population of unselected patients with atrial fibrillation who had not previously taken an oral anticoagulant. Patients were identified from nationwide registries in Denmark.

\section{Data sources}

In Denmark, individuals are assigned a unique identification number allowing linkage on an individual level between databases. Study data were obtained from three nationwide administrative databases: the Danish national prescription registry, which holds information on purchase date, Anatomical Therapeutic Chemical (ATC) classification code, and package size for every prescription claim since 1994; the Danish civil registration system, which holds information on sex, date of birth, and vital and emigration status; and the Danish national patient register, which includes admission/ discharge date, and discharge ICD (international classification of diseases) diagnosis codes for hospital admissions since 1977.

\section{Patient population}

Eligible patients were identified as those with a first time prescription claim for an NOAC defined as: apixaban (introduced 10 December 2012), dabigatran (introduced 1 August 2011), or rivaroxaban (introduced 1 February 2012), as well as individuals who started warfarin treatment (since 1 August 2011) up to 28 February 2016. Patients who had taken any oral anticoagulant within the previous year were excluded to establish a naive cohort. All NOACs were restricted to reduced doses approved for stroke prevention in atrial fibrillation (in Europe) as follows: apixaban $2.5 \mathrm{mg}$, dabigatran $110 \mathrm{mg}$, and rivaroxaban $15 \mathrm{mg}$. To focus on nonvalvular atrial fibrillation, we excluded patients with previous hospital diagnoses indicating valvular atrial fibrillation (mitral stenosis or mechanical heart valves). We further excluded all those patients with an indication for oral anticoagulant treatment other than atrial fibrillation (history of pulmonary embolism, deep venous thrombosis, or recent hip/knee surgery). This allowed for an indirect identification of eligible patients with atrial fibrillation as all other reasons for initiation of an oral anticoagulation were ruled out. We therefore assumed that initiation of treatment indicated stroke prophylaxis for atrial fibrillation.

\section{Patient involvement}

No patients were involved in setting the research question or the outcome measures, nor were they involved in developing plans for recruitment, design, or implementation of the study. No patients were asked to advise on interpretation or writing up of results. There are no plans to disseminate the results of the research to study participants or the relevant patient community.

\section{Ascertainment of outcome measures and comorbidity}

We used the Danish national patient register to follow included patients from onset of treatment until 30 April 2016 for the occurrence of a combined endpoint of "ischaemic stroke or systemic embolism" and ischaemic stroke separately. All cause mortality was included as a single endpoint because oral anticoagulants significantly reduce the risk of stroke and death compared with control or placebo, ${ }^{12}$ while NOACs significantly reduce all cause mortality compared with warfarin. ${ }^{13}$ Safety outcomes were recorded as haemorrhagic stroke and major and gastrointestinal bleeding and reported in a combined endpoint as "any bleeding” and specific for haemorrhagic stroke. Major bleeding was defined as bleeding with anaemia, haemothorax, haematuria, epistaxis, and bleeding in the eye (see table A in appendix 1). We used ICD-10 (international classification of diseases, 10th revision) to identify outcomes. The coding accuracy of the selected outcomes has previously been validated and found sufficiently accurate for epidemiological research. ${ }^{1415}$

For quantifying thromboembolic risk, we combined comorbidity information into the $\mathrm{CHA}_{2} \mathrm{DS}_{2}$-VASc score $^{16}$; similarly, to assess the risk of bleeding we calculated the HAS-BLED score (table B in appendix 2). ${ }^{17}$

Within each NOAC agent, there are differences in criteria for recommendation of a reduced dose; nevertheless, elderly people and those with renal impairment are subgroups of patients who require special attention regarding choice of NOAC and dose. ${ }^{18}$ Therefore, we analysed these subgroups as having a potential 
"indication for dose reduction." We identified those with adult polycystic kidney disease, chronic glomerulonephritis, chronic tubulointestinal nephropathy, diabetic nephropathy, non-end stage chronic kidney disease, hypertensive nephropathy, and nephropathy of unknown aetiology and categorised them as having chronic kidney disease (table A in appendix 1). While we did not have access to individual eGFR or creatinine clearance directly at the time of treatment initiation, a reasonable clinical assumption is that the aforementioned diseases would affect the renal function to such an extent that it could confer a clinical recommendation to reduce the dose of any NOAC.

\section{Statistical analyses}

Characteristics of the study population were reported as percentages, means (standard deviation), or medians (interquartile range). Person years of follow-up were calculated from the date of first prescription claim to the occurrence of first endpoint, death, emigration, or end of follow-up, whichever came first. Incidence rates were calculated as number of events divided by person time. To determine the risk of outcomes among NOAC users compared with warfarin (reference) users, we calculated cause specific hazard ratios using Cox regression models. Failure curves were used to depict how risks of events evolved over time. Specifically, we used the Aalen-Johansen estimator to calculate absolute risk of events taking into account the competing risk of death ${ }^{16}$ and the Kaplan-Meier estimator for all cause mortality. To allow an unbiased comparison across different treatment regimens, we applied an inverse probability of treatment weighted approach. This is particularly suitable when there are several treatment alternatives-in this case, dabigatran, apixaban, rivaroxaban, and warfarin. ${ }^{1920}$ The weights were derived to obtain estimates representing population average treatment effects with optimal balance between the treatment populations ${ }^{21}$ by using generalised boosted models based on 10000 regression trees as done previously. ${ }^{4}$ The underlying propensity models included age (continuous); binary indicators for sex and hospital diagnosis of atrial fibrillation, ischaemic stroke, vascular disease, ischaemic heart disease, hypertension, diabetes, cancer, chronic obstructive lung disease, heart failure or left ventricular dysfunction, recent prescription of aspirin, $\beta$ blockers, non-steroidal anti-inflammatory drugs, statins, loop and non-loop diuretics, amiodarone, dronedarone, vasodilators, calcium channel blockers, and verapamil; and the $\mathrm{CHA}_{2} \mathrm{DS}_{2}$-VASc and HAS-BLED scores included as continuous variables.

The examined treatment regimens should be contrasted on comparable populations and any patient must have positive probability for any treatment (positivity assumption), hence substantial overlap between the propensities for each treatment should be present. In agreement with best methodological practice, this was assessed by graphical inspection of the weight distributions (appendix 2). ${ }^{22}$ Additionally, we evaluated balance between treatment populations by standardised differences of all baseline covariates, using a threshold of 0.1 to indicate imbalance. ${ }^{23}$

\section{Sensitivity analyses}

To further explore the potential for bias from baseline differences and the propensity of receiving treatment with an NOAC, we performed sensitivity analyses using an ordinary crude and Cox multivariate adjusted analysis to compare the results obtained from the weighted analyses. We also performed a sensitivity analysis using standardised morbidity ratio ${ }^{24}$ weights to address the (hypothetical) casual situation of all patients receiving warfarin treatment rather than an NOAC.

To allow for a thorough prognostic evaluation and given the divergence of age across oral anticoagulant exposure groups, we supplemented the main analysis by a sensitivity analysis stratified on age category-for instance, age $\geq 80$. While not all of these patients would have renal deficiencies severe enough to acquire a diagnosis, advanced age significantly affects renal function $^{2526}$; thus, assessment of comparative effectiveness and safety between NOAC and warfarin in these age groups is of clinical value given the age dependency on renal function (opting for reduce dose NOAC regimen). We also conducted a sensitivity analysis restricted to patients with a hospital diagnosis of atrial fibrillation to increase likelihood of treatment indication. Finally, as the introduction of each NOAC could have influenced patient selection, we repeated the main analysis confined to the time period where all three NOACs were available in Denmark-that is, from 12 December 2012, when apixaban (latest market drug) was available in Denmark.

The analyses were performed with Stata version 14 (StataCorp) and R version 3.1.1 (R Foundation for Statistical Computing). A two sided $\mathrm{P}<0.05$ was considered significant.

\section{Results}

We identified 88141 patients in the study period who were eligible for inclusion by either fillinga prescription for low dose regimen NOAC or warfarin (only $2.5 \mathrm{mg}$ available in Denmark). After we excluded reasons for oral anticoagulant treatment other than atrial fibrillation $(n=31852)$ and previous use of phenprocoumon within the past year for unknown reasons $(n=645)$, we identified a study population $(n=55644)$ of patients who had not been taking oral anticoagulants within the previous year and who were starting treatment with either reduced dose NOAC or warfarin (fig A in appendix 3).

The study population was distributed by type of oral anticoagulant: $69.9 \%$ received warfarin, $7.9 \%$ apixaban, $15.9 \%$ dabigatran, and $6.3 \%$ rivaroxaban. The population average time of follow-up (with respect to all cause mortality) was 2.3 years, with the apixaban group having the shortest mean follow-up of one year. The study population age varied markedly across exposure to each drug-for example, the average age for apixaban users was 83.9, while for warfarin the average age was 71.0 (table 1). Patients treated with either apixaban or rivaroxaban had a higher prevalence of renal diseases 
(9.5\% and 9.1\%, respectively) than patients treated with dabigatran $(3.9 \%)$ or warfarin $(8.3 \%)$. In general, the patients treated with apixaban had more comorbidities such as heart failure, previous thromboembolism, diabetes, and presence of vascular diseases. Therefore, the estimated risk of stroke, as summarised by the mean $\mathrm{CHA}_{2} \mathrm{DS}_{2}$-VASc score, was highest in patients treated with apixaban (4.3), and slightly lower for dabigatran (3.8) and rivaroxaban (3.6), and lowest for warfarin treated patients (3.0). A similar pattern (but with less pronounced differences) for risk of bleeding, as summarised by the mean HASBLED score, was observed with an overall population average score of 2.4.

With inverse probability of treatment weight to account for baseline differences, the standardised differences were less than 0.09 (compared with 1.01 before imposing estimated weights). Comparisons of individual propensity score distributions showed sufficient overlap, and suggest that application of the weights of inverse probability of treatment, resulted in a cohort in which the distribution of variables were comparable between treatment groups; hence comparisons between treatment groups were feasible (appendix 2).

\section{Ischaemic stroke and systemic embolism}

A total of 1779 patients experience ischaemic stroke or systemic embolism events during the first year of follow-up (table 2). The highest weighted event rate was for apixaban (4.8\%) and the lowest for dabigatran (3.3\%), but similar to rivaroxaban (3.5\%) and warfarin (3.7\%) (table 2). Figure 1 shows the cumulative incidences for ischaemic stroke or systemic embolism across the four treatment options.

Apixaban was associated with a non-significant trend for higher rates of ischaemic stroke or systemic embolism compared with warfarin, with a one year hazard ratio of 1.19 (95\% confidence interval 0.95 to 1.49) (fig 2). The observed association after 2.5 years was 1.22 (1.00 to 1.50) (fig B in appendix 3). Rivaroxaban was associated with a non-significant trend towards lower rates after one year and 2.5 years of follow-up, with hazard ratios of 0.89 (0.69 to 1.16) and

\begin{tabular}{|c|c|c|c|c|c|c|c|}
\hline & \multirow{2}{*}{$\begin{array}{l}\text { Apixaban } 2.5 \\
\mathrm{mg} \text { twice/day } \\
(\mathrm{n}=4400)\end{array}$} & \multirow{2}{*}{$\begin{array}{l}\text { Dabigatran } \\
110 \text { mg twice/ } \\
\text { day }(\mathrm{n}=8875)\end{array}$} & \multirow{2}{*}{$\begin{array}{l}\text { Rivaroxaban } \\
15 \text { mg once/ } \\
\text { day }(n=3476)\end{array}$} & \multirow{2}{*}{$\begin{array}{l}\text { Warfarin } \\
(n=38893)\end{array}$} & \multirow[b]{2}{*}{ All $(n=55644)$} & \multicolumn{2}{|c|}{$\begin{array}{l}\text { Standardised } \\
\text { differences }^{*}\end{array}$} \\
\hline & & & & & & Before & After \\
\hline Women & $60.6(2665)$ & $53.7(4762)$ & $53.2(1848)$ & $40.4(15703)$ & 44.9 (24978) & 0.41 & 0.03 \\
\hline \multicolumn{8}{|l|}{ Age (years): } \\
\hline Mean (SD) & $83.9(8.2)$ & $79.9(9.0)$ & $77.9(13.5)$ & $71.0(12.6)$ & $73.9(12.7)$ & 1.01 & 0.09 \\
\hline$\geq 65$ & $97.2(4275)$ & $93.6(8309)$ & $85.7(2980)$ & $74.6(29001)$ & $80.1(44565)$ & - & - \\
\hline$\geq 75$ & $88.1(3878)$ & $78.1(6929)$ & $66.8(2321)$ & $41.3(16078)$ & $52.5(29206)$ & - & - \\
\hline$\geq 80$ & $75.3(3313)$ & $58.1(5159)$ & $53.5(1858)$ & $24.5(9523)$ & $35.7(19853)$ & - & - \\
\hline$\geq 85$ & $48.3(2124)$ & $28.4(2519)$ & $35.2(1222)$ & $11.1(4311)$ & $18.3(10176)$ & - & - \\
\hline Previous diagnosis of atrial fibrillation from hospital & $71.3(3135)$ & $64.8(5753)$ & $52.4(1821)$ & $55.4(21557)$ & $58.0(32266)$ & 0.38 & 0.06 \\
\hline Cancer & $22.2(976)$ & $18.3(1622)$ & $20.0(696)$ & $16.7(6508)$ & $17.6(9802)$ & 0.14 & 0.04 \\
\hline Vascular disease & $22.0(970)$ & $17.7(1570)$ & $18.2(631)$ & $19.0(7395)$ & $19.0(10566)$ & 0.11 & 0.03 \\
\hline Diabetes & $17.3(763)$ & $14.9(1321)$ & $16.5(575)$ & $16.3(6324)$ & $16.1(8983)$ & 0.07 & 0.02 \\
\hline Previous bleeding episodes & $17.3(761)$ & $14.3(1270)$ & $15.0(520)$ & $11.4(4422)$ & $12.5(6973)$ & 0.18 & 0.03 \\
\hline Hypertension & $63.5(2796)$ & $64.0(5676)$ & $58.1(2020)$ & $60.3(23447)$ & $61.0(33939)$ & 0.12 & 0.04 \\
\hline Previous ischaemic stroke & $22.9(1007)$ & $16.0(1423)$ & $15.2(528)$ & $11.0(4291)$ & $13.0(7249)$ & 0.35 & 0.02 \\
\hline Ischaemic heart disease & $29.9(1316)$ & $26.3(2337)$ & $26.7(929)$ & $26.8(10430)$ & $27.0(15.12)$ & 0.08 & 0.03 \\
\hline Heart failure/LVD & $20.3(892)$ & $15.5(1373)$ & $18.9(658)$ & $15.5(6024)$ & $16.1(8947)$ & 0.13 & 0.01 \\
\hline Mean (SD) $\mathrm{CHA}_{2} \mathrm{DS}_{2}$-VASC score & $4.3(1.5)$ & $3.8(1.5)$ & $3.6(1.8)$ & $3.0(1.7)$ & $3.3(1.7)$ & 0.75 & 0.04 \\
\hline Mean (SD) HASBLED score & $2.8(1.1)$ & $2.7(1.0)$ & $2.5(1.2)$ & $2.4(1.2)$ & $2.4(1.2)$ & 0.41 & 0.06 \\
\hline Chronic obstructive pulmonary disease & $18.3(803)$ & 14.9 (1319) & $16.7(579)$ & $13.0(5051)$ & $13.9(7752)$ & 0.15 & 0.03 \\
\hline Dialysis & $0.9(39)$ & $0.5(41)$ & $0.9(30)$ & $2.4(920)$ & $1.9(1030)$ & 0.14 & 0.05 \\
\hline Renal dysfunction & $9.5(417)$ & $3.9(350)$ & $9.1(315)$ & $8.3(3244)$ & $7.8(4326)$ & 0.21 & 0.04 \\
\hline Aspirin & $48.2(2122)$ & $50.3(4460)$ & $44.4(1545)$ & $46.8(18183)$ & $47.3(26310)$ & 0.12 & 0.05 \\
\hline$\beta$ blocker & $60.0(2639)$ & $62.1(5513)$ & $50.5(1755)$ & $63.0(24515)$ & $61.9(34422)$ & 0.26 & 0.03 \\
\hline Non-steroidal anti-inflammatory drugs & $18.5(813)$ & $24.5(2172)$ & $21.8(758)$ & $24.4(9471)$ & $23.7(13214)$ & 0.14 & 0.02 \\
\hline Statins & $42.5(1871)$ & $43.5(3861)$ & $40.4(1403)$ & $45.0(17488)$ & $44.3(24623)$ & 0.09 & 0.01 \\
\hline Loop diuretics & $43.2(1902)$ & $32.3(2865)$ & $38.3(1333)$ & $29.8(11603)$ & $31.8(17703)$ & 0.29 & 0.04 \\
\hline Non-loop diuretics & $40.8(1794)$ & 44.1 (3913) & $39.0(1354)$ & 39.5 (15375) & $40.3(22436)$ & 0.11 & 0.02 \\
\hline Amiodarone & $4.3(189)$ & $3.5(312)$ & $3.4(119)$ & $4.5(1731)$ & $4.2(2351)$ & 0.05 & 0.02 \\
\hline Dronedarone & $<5$ & $<5$ & $<5$ & $<5$ & $<5$ & 0.05 & 0.04 \\
\hline Vasodilator & $4.5(198)$ & $4.7(417)$ & $5.0(174)$ & $4.5(1768)$ & $4.6(2557)$ & 0.02 & 0.03 \\
\hline Calcium blockers & $33.8(1486)$ & $35.6(3160)$ & 30.5 (1059) & $33.1(12893)$ & $33.4(18598)$ & 0.11 & 0.01 \\
\hline Verapamil & $3.0(130)$ & $5.1(457)$ & $2.7(94)$ & $3.4(1327)$ & $3.6(2008)$ & 0.13 & 0.05 \\
\hline P-glycoprotein inhibitors & $8.9(391)$ & $10.3(914)$ & $8.1(281)$ & $9.6(3753)$ & $9.6(5339)$ & 0.08 & 0.04 \\
\hline CYP3A4 inhibitors & $3.8(165)$ & $2.6(231)$ & $3.6(124)$ & $3.0(1173)$ & 3.0 (1693) & 0.07 & 0.02 \\
\hline
\end{tabular}


Table 2 | Event counts and crude and weighted event rates per 100 person years among patients with atrial fibrillation who had not previously received oral anticoagulant by treatment groups

\begin{tabular}{|c|c|c|c|c|c|c|c|c|c|c|c|c|}
\hline \multirow[b]{2}{*}{ Outcomes } & \multicolumn{3}{|c|}{ Apixaban $2.5 \mathrm{mg}$} & \multicolumn{3}{|c|}{ Dabigatran $110 \mathrm{mg}$} & \multicolumn{3}{|c|}{ Rivaroxaban $15 \mathrm{mg}$} & \multicolumn{3}{|c|}{ Warfarin } \\
\hline & Events & Crude & Weighted & Events & Crude & Weighted & Events & Crude & Weighted & Events & Crude & Weighted \\
\hline \multicolumn{13}{|l|}{ One year follow-up } \\
\hline Ischaemic stroke/systemic embolism & 205 & 6.67 & 4.78 & 325 & 4.11 & 3.31 & 92 & 3.55 & 3.53 & 1157 & 3.31 & 3.74 \\
\hline Ischaemic stroke & 198 & 6.44 & 4.42 & 309 & 3.90 & 3.17 & 88 & 3.39 & 3.38 & 1059 & 3.02 & 3.47 \\
\hline All cause mortality & 806 & 25.40 & 15.53 & 985 & 12.18 & 10.50 & 557 & 21.15 & 15.81 & 3048 & 8.56 & 10.12 \\
\hline Any bleeding & 176 & 5.67 & 5.12 & 377 & 4.76 & 4.09 & 169 & 6.60 & 5.58 & 1759 & 5.06 & 5.11 \\
\hline Major bleeding & 123 & 3.95 & 4.14 & 291 & 3.66 & 3.31 & 135 & 5.24 & 4.59 & 1281 & 3.67 & 3.82 \\
\hline Haemorrhagic stroke & 22 & 0.69 & 0.38 & 28 & 0.35 & 0.28 & 10 & 0.38 & 0.43 & 199 & 0.56 & 0.62 \\
\hline \multicolumn{13}{|l|}{2.5 year follow-up } \\
\hline Ischaemic stroke/systemic embolism & 236 & 5.63 & 3.98 & 535 & 3.26 & 2.73 & 124 & 2.89 & 2.72 & 1686 & 2.35 & 2.68 \\
\hline Ischaemic stroke & 230 & 5.48 & 3.73 & 504 & 3.06 & 2.58 & 119 & 2.77 & 2.62 & 1558 & 2.17 & 2.49 \\
\hline All cause mortality & 1040 & 23.80 & 14.85 & 1873 & 11.02 & 9.13 & 798 & 18.18 & 13.46 & 5366 & 7.29 & 8.73 \\
\hline Any bleeding & 224 & 5.29 & 4.76 & 659 & 4.03 & 3.44 & 240 & 5.72 & 4.90 & 2910 & 4.12 & 4.16 \\
\hline Major bleeding & 160 & 3.75 & 3.90 & 491 & 2.98 & 2.78 & 187 & 4.42 & 3.97 & 2136 & 3.00 & 3.14 \\
\hline Haemorrhagic stroke & 27 & 0.62 & 0.35 & 68 & 0.40 & 0.30 & 26 & 0.59 & 0.56 & 336 & 0.46 & 0.50 \\
\hline
\end{tabular}

Crude failure curves

Ischaemic stroke or systemic embolism
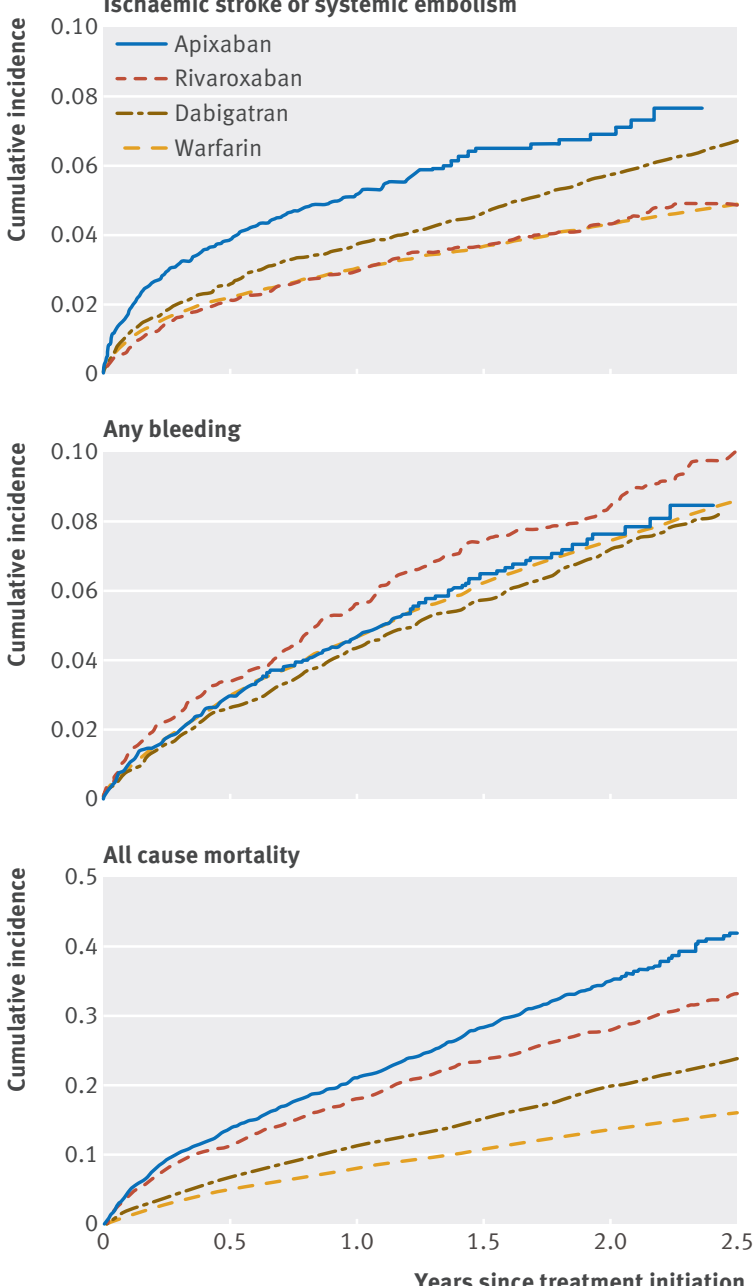

Weighted failure curves
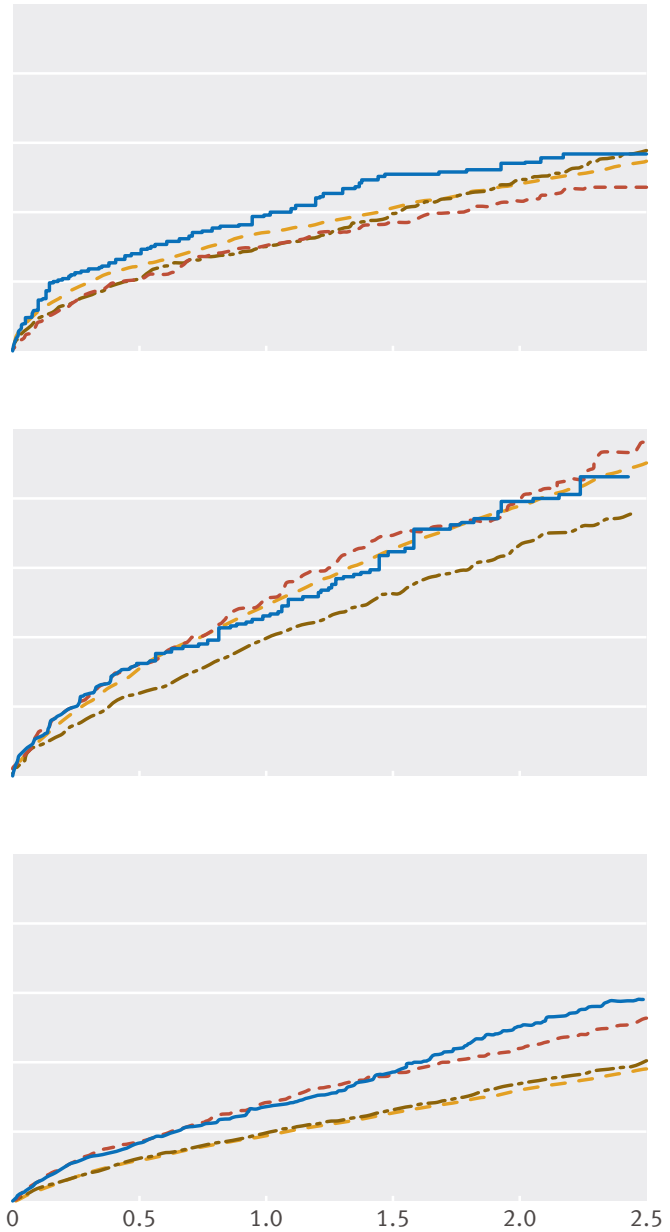

Years since treatment initiation

Fig 1 Cumulative risk of events depicted by crude and weighted failure curves in patients with atrial fibrillation according to initiated treatment 
Ischaemic stroke/SE

\begin{tabular}{|c|c|c|}
\hline \multirow{2}{*}{\multicolumn{2}{|c|}{$\begin{array}{lc}\text { Study } & \text { Hazard ratio } \\
& (95 \% \mathrm{CI}) \\
\text { Entire cohort, analysis weighted for inverse } \\
\text { probability of treatment }\end{array}$}} & \multirow[t]{2}{*}{$\begin{array}{l}\text { Hazard ratio } \\
\quad(95 \% \mathrm{Cl})\end{array}$} \\
\hline & & \\
\hline Apixaban & 1.19 (0.95 to 1.49$)$ & \\
\hline Dabigatran & 0.89 (0.77 to 1.03$)$ & $\Rightarrow$ \\
\hline Rivaroxaban & 0.89 (0.69 to 1.16$)$ & \\
\hline \multicolumn{3}{|c|}{ Indication for reduced dose (additional analysis) } \\
\hline Apixaban & 1.24 (1.00 to 1.55$)$ & \\
\hline Dabigatran & 0.93 (0.79 to 1.10$)$ & \\
\hline Rivaroxaban & 0.63 (0.47 to 0.85$)$ & $\longrightarrow$ \\
\hline \multicolumn{3}{|c|}{ Entire cohort (adjusted analysis) } \\
\hline Apixaban & 1.07 (0.91 to 1.26$)$ & \\
\hline Dabigatran & 0.94 (0.82 to 1.07$)$ & \\
\hline Rivaroxaban & 0.78 (0.63 to 0.97$)$ & \\
\hline \multicolumn{3}{|c|}{ Age $\geq 80$ (sensitivity analysis) } \\
\hline Apixaban & 1.15 (0.94 to 1.41$)$ & \\
\hline Dabigatran & 0.98 (0.82 to 1.17$)$ & \\
\hline Rivaroxaban & $0.71(0.52$ to 0.95$)$ & - \\
\hline \multicolumn{3}{|c|}{$\begin{array}{l}\text { Cohort with atrial fibrillation diagnosed in } \\
\text { hospital (sensitivity analysis) }\end{array}$} \\
\hline Apixaban & $1.06(0.81$ to 1.40$)$ & \\
\hline Dabigatran & 0.91 (0.77 to 1.07$)$ & \\
\hline \multirow[t]{3}{*}{ Rivaroxaban } & 0.98 (0.71 to 1.34$)$ & \\
\hline & & 0.5 \\
\hline & \multicolumn{2}{|c|}{ Favours } \\
\hline
\end{tabular}

Ischaemic stroke

Hazard ratio
$(95 \% \mathrm{Cl})$

1.19 (0.95 to 1.49$)$

0.92 (0.79 to 1.06$)$

0.93 (0.71 to 1.21 )

1.25 (1.00 to 1.57$)$

0.93 ( 0.78 to 1.11$)$

0.64 ( 0.47 to 0.87 )

1.07 (0.90 to 1.26$)$

0.94 (0.82 to 1.07$)$

0.79 ( 0.63 to 0.99$)$

1.18 (0.96 to 1.45$)$

0.97 (0.81 to 1.16$)$

0.71 ( 0.52 to 0.96$)$

1.03 (0.78 to 1.36$)$

0.91 (0.76 to 1.08 )

1.00 (0.72 to 1.38$)$
Hazard ratio $(95 \% \mathrm{Cl})$

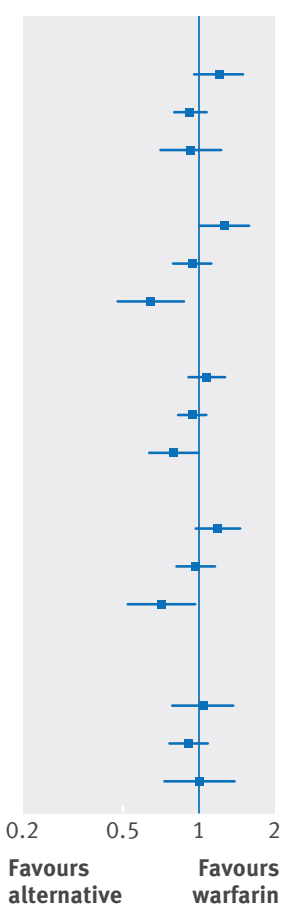

Death

$\begin{array}{cc}\text { Hazard ratio } & \text { Hazard ratio } \\ (95 \% \mathrm{Cl}) & (95 \% \mathrm{Cl})\end{array}$

1.48 (1.31 to 1.67$)$

1.04 (0.96 to 1.13 )

1.52 (1.36 to 1.70 )

1.23 (1.10 to 1.36$)$

0.93 (0.84 to 1.02 )

1.48 (1.32 to 1.67 )

1.35 (1.24 to 1.47$)$

1.03 (0.96 to 1.11$)$

1.43 (1.30 to 1.57$)$

1.54 (1.40 to 1.70$)$

$1.00(0.91$ to 1.10$)$

1.67 (1.49 to 1.87 )

1.57 (1.37 to 1.79$)$

1.07 (0.97 to 1.19$)$

1.48 (1.29 to 1.71$)$

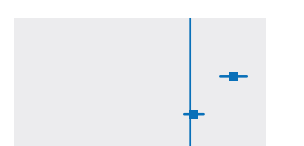

Fig 2 | Effectiveness outcomes at one year follow-up in patients with atrial fibrillation according to initiated treatment
0.92 (0.73 to 1.15$)$, respectively. For dabigatran there was a non-significant trend towards lower rates in comparison with warfarin after one year $(0.89,0.77$ to 1.03), and similar to warfarin after 2.5 years of follow-up (1.03, 0.92 to 1.16). Restriction of the outcome to lone ischaemic stroke did not markedly affect the event rate (fig 2).

\section{Bleeding outcomes}

The weighted event rates for bleeding outcomes were similar for apixaban, rivaroxaban, and warfarin at 5.1\%, $5.6 \%$, and $5.1 \%$, respectively, and lower for dabigatran (4.1\%). For the composite outcome of any bleeding events, the cumulative incidence curves were similar for apixaban, rivaroxaban, and warfarin, while lower risks were observed for dabigatran (fig 1).

Weighted Cox regression comparisons showed lower event rates for dabigatran compared with warfarin after one and 2.5 years (hazard ratios 0.80 (95\% confidence interval 0.70 to 0.92 ) (fig 3) and 0.84 (0.75 to 0.93) (fig C in appendix 3), respectively). The one year hazard ratios for bleeding for apixaban and rivaroxaban compared with warfarin were 0.96 (0.73 to 1.27$)$ and 1.06 (0.87 to 1.29), respectively. Examination of bleeding outcomes during 2.5 years of follow-up showed similar associations (fig $\mathrm{C}$ in appendix 3).

Analysis of the major bleeding outcome showed similar associations to the composite "any bleeding" outcome, except for dabigatran, which was associated with a non-significant decrease in event rate compared with warfarin, with a hazard ratio of 0.87 ( $95 \%$ confidence interval 0.75 to 1.01 ).
During one year of follow-up, there was a limited number of haemorrhagic strokes: 60 with NOAC and 199 with warfarin (table 2 ). The one year event rates for haemorrhagic stroke were lower for patients treated with a NOAC agent compared with warfarin, with hazard ratios ranging from 0.46 to 0.68 (fig 3). After 2.5 years, only hazard ratios for dabigatran were significantly lower in comparison with warfarin, while an association of similar rates was observed with rivaroxaban and warfarin.

\section{Death}

The risk of all cause mortality was different between NOACs and warfarin, with higher risks for patients treated with apixaban and rivaroxaban. The crude one year mortality risk was $8.6 \%$ for warfarin, $12.2 \%$ for dabigatran, $21.2 \%$ for rivaroxaban, and $25.4 \%$ for apixaban (table 2). After we weighted the treatment cohorts, the differences in risks were attenuated (fig 1). Hazard ratios were then 1.48 (95\% confidence interval 1.31 to 1.67) for apixaban, 1.04 ( 0.96 to 1.13) for dabigatran; and 1.52 (1.36 to 1.70) for rivaroxaban (fig 2). The associations remained similar when we analysed risk of death during 2.5 years of follow-up (fig B in appendix 3).

\section{Indication for dose reduction according to age and renal disease}

When we analysed the cohort stratified according to "indication for dose reduction" (that is, age $\geq 80$ and/or renal disease) the study population was confined to 21949 patients. During the period 2014-16, apixaban 2.5 $\mathrm{mg}$ was increasingly prescribed to patients in this subgroup, while the other three treatment options were 


\section{Any bleeding}

\section{Study}

Hazard ratio $(95 \% \mathrm{Cl})$

Entire cohort, analysis weighted for inverse probability of treatment

\begin{tabular}{|c|c|c|c|}
\hline & \multirow{2}{*}{\multicolumn{2}{|c|}{$\rightarrow$}} \\
\hline Apixaban & $0.96(0.73$ to 1.27$)$ & & \\
\hline Dabigatran & $0.80(0.70$ to 0.92$)$ & - & \\
\hline Rivaroxaban & $1.06(0.87$ to 1.29$)$ & & \\
\hline \multicolumn{4}{|c|}{ ndication for reduced dose (additional analysis) } \\
\hline Dabigatran & $0.81(0.69$ to 0.94$)$ & $\Rightarrow$ & \\
\hline Rivaroxaban & $1.00(0.81$ to 1.24$)$ & . & \\
\hline \multicolumn{4}{|c|}{ Entire cohort (adjusted analysis) } \\
\hline Apixaban & $0.76(0.64$ to 0.90$)$ & $=-$ & \\
\hline Dabigatran & $0.81(0.72$ to 0.91$)$ & - & \\
\hline Rivaroxaban & $1.07(0.91$ to 1.26$)$ & & \\
\hline \multicolumn{4}{|c|}{ Age $\geq 80$ (sensitivity analysis) } \\
\hline Apixaban & 0.78 (0.63 to 0.96$)$ & $\rightarrow$ & \\
\hline Dabigatran & $0.89(0.76$ to 1.04$)$ & $\Rightarrow$ & \\
\hline Rivaroxaban & $1.13(0.91$ to 1.40$)$ & & $=-$ \\
\hline \multicolumn{4}{|c|}{$\begin{array}{l}\text { Cohort with atrial fibrillation diagnosed in } \\
\text { hospital (sensitivity analysis) }\end{array}$} \\
\hline Apixaban & 0.95 (0.68 to 1.33$)$ & - & \\
\hline Dabigatran & $0.81(0.68$ to 0.96$)$ & $\rightarrow-$ & \\
\hline \multirow[t]{2}{*}{ Rivaroxaban } & 1.05 (0.81 to 1.36$)$ & & \\
\hline & 0.2 & 0.5 & \\
\hline
\end{tabular}

Major bleeding

Hazard ratio $(95 \% \mathrm{Cl})$

1.04 (0.76 to 1.43$)$

0.87 (0.75 to 1.01$)$

1.17 (0.94 to 1.45$)$

0.73 (0.57 to 0.94$)$

0.88 (0.75 to 1.04$)$

1.13 (0.89 to 1.44$)$

0.79 (0.65 to 0.96$)$

0.89 (0.78 to 1.02$)$

1.19 (0.99 to 1.43$)$

0.85 (0.67 to 1.08$)$

$1.01(0.84$ to 1.20$)$

1.28 (1.00 to 1.63$)$

1.02 (0.69 to 1.52$)$

0.91 (0.75 to 1.10$)$

$1.13(0.85$ to 1.51$)$

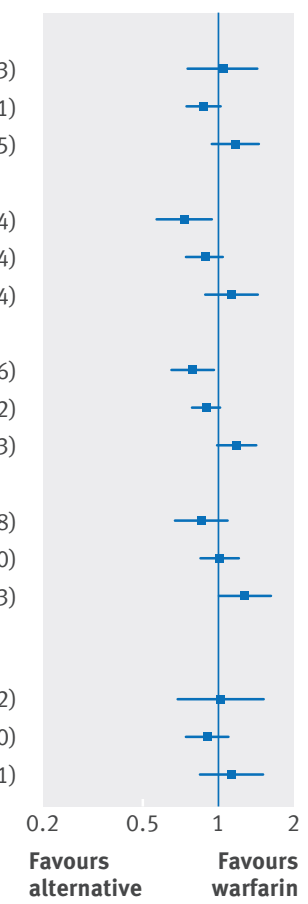
$(95 \% \mathrm{Cl})$
Haemorrhagic stroke

Hazard ratio (95\% CI) (95\%
0.59 (0.34 to 1.02$)$

$0.46(0.29$ to 0.72$)$

0.68 (0.30 to 1.53$)$

$0.84(0.47$ to 1.50$)$

0.49 (0.27 to 0.87$)$

$0.46(0.20$ to 1.04$)$

0.83 (0.52 to 1.33$)$

0.47 (0.31 to 0.71$)$

$0.56(0.29$ to 1.06$)$

0.82 (0.46 to 1.45$)$

0.46 (0.27 to 0.78$)$

$0.54(0.23$ to 1.29$)$

0.56 (0.29 to 1.05$)$

0.29 (0.16 to 0.50 )

0.79 (0.31 to 2.05 )

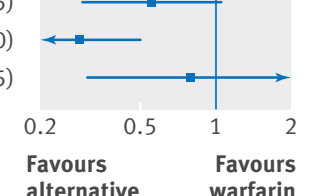

Fig 3 | Safety outcomes at one year follow-up in patients with atrial fibrillation according to initiated treatment

prescribed less often (fig D in appendix 3). This more fragile cohort exhibited worse outcomes than we saw in the main analysis.

For the outcome of ischaemic stroke or systemic embolism, apixaban was associated with a higher one year rate compared with warfarin (hazard ratio 1.24 , 95\% confidence interval 1.00 to 1.55 ) (fig 2), and rivaroxaban was associated with lower rates (0.63, 0.47 to 0.85 ). For the composite bleeding outcome, both apixaban and dabigatran had lower rates compared with warfarin (0.78, 0.61 to 0.99 , and $0.81,0.69$ to 0.94 , respectively). Rivaroxaban had similar rate of bleeding compared with warfarin (1.00, 0.81 to 1.24) (fig 3). Results on patients with renal disease yield comparable conclusion (fig E in appendix 3).

For the outcome of all cause mortality, apixaban and rivaroxaban was associated with a significantly higher risk (hazard ratios 1.23 , 95\% confidence interval 1.10 to 1.36 , and $1.48,1.32$ to 1.67 , respectively). For dabigatran there was no significant difference $(0.93,0.84$ to 1.02$)$.

\section{Sensitivity analyses}

In the treatment cohorts confined to those aged $\geq 80$ for the event rate of ischaemic stroke or systemic embolism, apixaban was associated with a non-significant trend for higher rates comparison with warfarin (one year hazard ratio 1.15 , 95\% confidence interval 0.94 to 1.41 ) (fig 2). The event rate was lower for rivaroxaban (0.71, 0.52 to 0.95 ) and similar for dabigatran and warfarin (0.98, 0.82 to 1.17$)$. For bleeding outcomes, the one year hazard ratios for NOACs compared with warfarin were
0.78 (95\% confidence interval 0.63 to 0.96 ) for apixaban, 0.89 ( 0.76 to 1.04 ) for dabigatran, and not significantly different for rivaroxaban (1.13, 0.91 to 1.40 ) (fig 3).

When we examined the outcomes in a cohort restricted to patients with a record of atrial fibrillation diagnosed in hospital, the outcomes associated with NOAC treatment compared with warfarin generally showed similarity in both effectiveness and safety outcomes, but dabigatran was associated with a lower relative risk of bleeding.

Our main analysis approach using the inverse probability treatment weight method was in overall agreement with the Cox multivariable adjusted analyses (figs 2 and 3), as well as the sensitivity analyses using a standardised morbidity ratio weighted approach (data not shown). When we confined the inclusion time from December 2012 (when apixaban became available) to February 2016, we did not observe markedly different associations between exposure and outcomes, except for dabigatran, which was not significantly different to warfarin for ischaemic stroke/systemic embolism (hazard ratio $1.04,95 \%$ confidence interval 0.86 to 1.25 ).

\section{Discussion}

This study examined the effectiveness and safety of low dose NOAC regimens compared with warfarin in a large "real world" cohort of patients with atrial fibrillation who had not previously received treatment with an oral anticoagulant. Treatments included apixaban $2.5 \mathrm{mg}$, dabigatran $110 \mathrm{mg}$, or rivaroxaban $15 \mathrm{mg}$ all compared with warfarin. Apixaban was associated with a 
non-significant trend towards higher thromboembolic events, with similar rates of rates of bleeding. Rivaroxaban was associated with a non-significant trend towards lower one year rates of ischaemic stroke or systemic embolism, with similar rates of rates of bleeding. Dabigatran was associated with lower rates of bleeding, with a non-significant trend for a lower thromboembolic rate after one year, though this trend was not seen after 2.5 years of follow-up.

Differences in reduced dose and standard dose NOAC Our study extends our previous finding in a similar cohort of patients with atrial fibrillation starting treatment with an NOAC at a standard dose (apixaban $5 \mathrm{mg}$, dabigatran $150 \mathrm{mg}$, rivaroxaban $20 \mathrm{mg}$ ) or warfarin. ${ }^{4}$ One major discrepancy between the studies was apparent when we analysed the outcome of all cause mortality. While the standard dosing regimen consistently favoured an NOAC agent over warfarin in terms of lower risk of death, the present study shows some differences in mortality between reduced dose regimens. Rivaroxaban was associated with an increase in risk of mortality in the main analysis, whereas dabigatran was associated with a decrease. In all additional and sensitivity analyses, both rivaroxaban and apixaban exhibited associations favouring warfarin in relation to an increase in risk of mortality. Although we performed inverse probability treatment weight analyses and accompanied this approach with standardised morbidity ratio analyses, the obtained associations from the main analysis remained unchanged. This might indicate that some residual confounding remained present and that this particular observation cannot rule out selective prescribing not adjusted for in the analyses. In particularly, this could be related to differences in population age across treatment groups. The results of sensitivity analyses specifically on age groups, however, did not differentiate markedly from the main analysis.

\section{Perspectives to randomised trial data}

In the Apixaban for Reduction in Stroke and Other Thromboembolic Events in Atrial Fibrillation (ARISTOTLE) trial, 831 patients received apixaban $2.5 \mathrm{mg}$ (with 428 patients initially randomised to this dose at baseline) based on at least two of age $\geq 80$, bodyweight $\leq 60$ $\mathrm{kg}$, and serum creatinine $\geq 132.6 \mu \mathrm{mol} / \mathrm{L} .{ }^{27}$ In a post hoc analysis on the ARISTOTLE trial data, 790 patients were treated with either apixaban $2.5 \mathrm{mg}$ or warfarin; all were aged $\geq 75$, but no additional information on indication for dose reduction or distribution of patients was given. ${ }^{28}$ In this subgroup of patients, the outcome of ischaemic stroke or systemic embolism occurred at a lower rate in the apixaban arm than in the warfarin arm (hazard ratio 0.52, 95\% confidence interval 0.25 to 1.08 ). As no information on dose reduction was provided, direct comparisons with the results in the current study would be challenging. Even so, in the subgroup of patients with an indication for dose reduction (age $\geq 80$ and/or renal dysfunction) we observed a higher rate of ischaemic stroke or systemic embolism with apixaban $2.5 \mathrm{mg}$ compared with warfarin $(1.24,1.00$ to 1.55$)$. Whether this discrepancy between post hoc trial analysis and data from routing care can be related to inappropriate prescribing patterns of apixaban $2.5 \mathrm{mg}$ in clinical practice remains to be investigated. Nevertheless, in healthy people (with normal renal function and lower age) apixaban $2.5 \mathrm{mg}$ was associated with about $50 \%$ lower plasma concentrations than treatment with $5.0 \mathrm{mg} .{ }^{29}$ Our observational data might raise the question of whether a 50\% dose reduction is excessive or appropriate in patients aged $\geq 80$ and/or with impaired renal function to maintain effective stroke prevention. Clearly, this hypothesis warrants further research.

Dabigatran $110 \mathrm{mg}$ was the only one of the three NOACs included in the present study that was formally analysed compared with warfarin in the landmark NOAC trials. ${ }^{30}$ Our observations support the findings from the Randomized Evaluation of Long-Term Anticoagulation Therapy (RE-LY) trial, which showed a hazard ratio of 0.91 (95\% confidence interval 0.74 to 1.11) for ischaemic stroke or systemic embolism for dabigatran $110 \mathrm{mg}$ compared with warfarin. Similarly, our observations were in line with the trial results when we analysed the outcome of major bleeding events. In a subset (post hoc) analysis of the RE-LY trial in patients with impaired renal function (as indicated by a creatinine clearance $<50 \mathrm{~mL} / \mathrm{min}\left(0.84 \mathrm{~mL} / \mathrm{s} / \mathrm{m}^{2}\right), 1196$ patients received dabigatran $110 \mathrm{mg}$ and 1232 patients received warfarin treatment. Patients in the dabigatran $110 \mathrm{mg}$ arm had a trend towards lower rates of ischaemic stroke or systemic embolism compared with those in the warfarin group, while the rates of major bleeding were similar between the two groups. ${ }^{31}$ In our data reflecting clinical practice, however, this trend of lower thromboembolic rates (in comparison with warfarin) was not observed after 2.5 years of follow-up.

In the ROCKET-AF trial (Rivaroxaban Once-daily, oral direct factor Xa inhibition compared with vitamin $\mathrm{K}$ antagonism for prevention of stroke and Embolism Trial in Atrial Fibrillation), 2959 patients had a creatinine clearance $<50 \mathrm{~mL} / \mathrm{min}$, with 1474 patients randomised to rivaroxaban $15 \mathrm{mg} .{ }^{3233}$ The median age in this subgroup was 79. There was a trend for lower rates of ischaemic stroke or systemic embolism with rivaroxaban 15 mg compared with warfarin in the ROCKET-AF trial, with similar rates of bleeding between the groups. Our data support the trial data with a trend of lower thromboembolic rates, which in the present study were also significantly lower compared with warfarin (specifically in the main analysis and in the analysis of "indication for dose reduction"; fig 2).

Balancing thromboembolism against the risk of bleeding in patients with atrial fibrillation is a clinical dilemma that involves more than scientific facts. Some of the factors include physician preferences and experience, patient's preferences and adherence to treatment, and cost and convenience related to the lifelong treatment. ${ }^{34-36}$ Nevertheless, the now historical trials in stroke prevention showed that treatment with an oral anticoagulant compared with placebo or control in 
patients with atrial fibrillation reduced the risk of ischaemic stroke as well as all cause death. ${ }^{12}$ Along these lines, it might be important to review concerns about safety of oral anticoagulant treatment in atrial fibrillation: ineffective or insufficient treatment for stroke prevention should be viewed as a safety issue itself, while the increase in the risk of bleeding is an inevitable consequence of a necessary treatment. Thus, choosing the appropriate antithrombotic agent for each individual is paramount to reduce the stroke burden in atrial fibrillation, while a relative increase in risk of bleeding cannot be ruled out. ${ }^{37}$

\section{Strengths and limitations}

The strength of this study primarily relates to the large sample size of patients with atrial fibrillation who had not previously received treatment with an oral anticoagulant and who were starting such treatment on a reduced dose regimen. Reduced dose apixaban and rivaroxaban were not formally investigated in the phase III trials (while in the RE-LY trial, patients were randomised to dabigatran $110 \mathrm{mg}$ or $150 \mathrm{mg}$ ). Our study complements this current evidence but is based on observational data and should be interpreted in this light. While inverse probability treatment weighting allows for causal inference on average treatment effects, it is likely that some unmeasured residual confounding and selective prescribing behaviour were still present. Specifically, quality of treatment in associations with social status and socioeconomic factors could have contributed to differences in outcomes associated with treatments. The overall obtained results were unchanged when we used simple adjustment or standardised morbidity ratio weighted approaches; this is reassuring and could suggest that modelling assumptions (including positivity, exchangeability, and consistency) were not violated. While these assumptions were not systematically investigated, the sample size of our data would suggest that the inverse probability treatment weight estimator is likely to be unbiased (contrary, when data are sparse, the estimator can be biased). ${ }^{38}$

Nonetheless, future studies on reduced dose NOAC versus warfarin are still warranted and should preferably analyse effectiveness and safety outcomes in respect to label adherence as done in a post hoc analyses with the RE-LY trial data. ${ }^{39}$ The lack of data on creatinine clearance is one of the limitations to the present study, even though patients with impaired renal function were identified in the registries. Indeed, worsening renal function in relation to age has been specifically investigated with data from the ARISTOTLE trial. In this cohort, elderly patients and those with accumulated cardiovascular comorbidities (apart from atrial fibrillation) more often had declining renal function. ${ }^{40}$ While the use of registries to identify patients with renal impairment has previously been reported as difficult, ${ }^{41}$ the patients with a record of kidney disease in our database had a clinical diagnosis and were therefore likely to be correctly classified. Nevertheless, we probably underestimated the true proportion of patients with renal impairment, and the interpretation of the results should accommodate this limitation. The differences in risk of death across treatment groups also warrant further investigation as to whether these results reflect "true" associations or biases from selective prescribing. As for now, these results should be confirmed in future studies, and our observations on associations between treatment with death cannot stand alone.

Finally, we cannot rule out the risk of misclassification and miscoding of diagnoses and outcomes; limitations previously noted by Schneeweiss and colleagues regarding comparative effectiveness also apply to our study. ${ }^{42}$ Observational studies, like the present one, however, give us the opportunity to ascertain how NOACs perform in clinical practice under usual clinical conditions.

\section{Conclusions}

In this propensity weighted nationwide study of reduced dose NOAC regimens, rates of ischaemic stroke or systemic embolism with apixaban $2.5 \mathrm{mg}$ were higher but not significantly different to those with warfarin. Though there was a trend towards lower thromboembolic rates with dabigatran $110 \mathrm{mg}$ and rivaroxaban 15 $\mathrm{mg}$, they were not significantly different to rates with warfarin. For the principal safety outcome, rates of bleeding were not significantly different with apixaban and rivaroxaban compared with warfarin but were significantly lower with dabigatran.

Contributors: All authors contributed to the design, analysis, interpretation of data, drafting the article, or revising it critically for important intellectual content and approved the final version to be published. TBL and GYHL are joint senior authors. PBN is guarantor. Funding: The Obel Family Foundation partly funded this research by an unrestricted grant. The sponsor had no role the design and conduct of the study; collection, management, analysis, and interpretation of the data; and preparation, review, or approval of the manuscript.

Competing interests: All authors have completed the ICMJE uniform disclosure form at www.icmje.org/coi_disclosure.pdf and declare: support from the Obel Family Foundation for the submitted work; TBL has served as an investigator for Janssen Scientific Affairs, LLC, and Boehringer Ingelheim and has served as a speaker for Bayer, BMS/ Pfizer, and Boehringer Ingelheim; PBN has served as a speaker for Boehringer Ingelheim and consultant for Bayer and received unrestricted research grant from BMS/Pfizer; GYHL has served as a consultant for Bayer, Astellas, Merck, Sanofi, BMS/Pfizer, DaiichiSankyo, Biotronik, Portola, and Boehringer Ingelheim and as a speaker for Bayer, BMS/Pfizer, Boehringer Ingelheim, Daiichi-Sankyo, and Sanofi Aventis; FS has served as a consultant for Bayer.

Ethical approval: Not required.

Data sharing: Data sharing is not possible because of legislation from the Danish Government.

Transparency: The lead author (PBN) affirms that the manuscript is an honest, accurate, and transparent account of the study being reported; that no important aspects of the study have been omitted and that any discrepancies from the study as planned (and, if relevant, registered).

This is an Open Access article distributed in accordance with the Creative Commons Attribution Non Commercial (CC BY-NC 4.0) license, which permits others to distribute, remix, adapt, build upon this work non-commercially, and license their derivative works on different terms, provided the original work is properly cited and the use is noncommercial. See: http://creativecommons.org/licenses/by-nc/4.0/.

1 Ruff CT, Giugliano RP, Braunwald E, et al. Comparison of the efficacy and safety of new oral anticoagulants with warfarin in patients with atrial fibrillation: a meta-analysis of randomised trials. Lancet 2014;383:955-62. doi:10.1016 S0140-6736(13)62343-0. 
2 January CT, Wann LS, Alpert JS, et al. American College of Cardiology/ American Heart Association Task Force on Practice Guidelines. 2014 AHA/ ACC/HRS guideline for the management of patients with atrial fibrillation: a report of the American College of Cardiology/American Heart Association Task Force on Practice Guidelines and the Heart Rhythm Society. J Am Coll Cardiol 2014;64:e1-76. doi:10.1016/j.jacc.2014.03.022.

3 Camm AJ, Lip GYH, De Caterina R, et al. ESC Committee for Practice Guidelines-CPG Document Reviewers. 2012 focused update of the ESC Guidelines for the management of atrial fibrillation: an update of the 2010 ESC Guidelines for the management of atrial fibrillation--developed with the special contribution of the European Heart Rhythm Association. Europace 2012;14:1385-413. doi:10.1093/europace/eus305.

4 Larsen TB, Skjøth F, Nielsen PB, Kiældgaard JN, Lip GYH. Comparative effectiveness and safety of non-vitamin $\mathrm{K}$ antagonist ora anticoagulants and warfarin in patients with atrial fibrillation: propensity weighted nationwide cohort study. BMJ 2016;353:i3189. doi:10.1136/bmi.i3189.

5 Larsen TB, Rasmussen LH, Skjøth F, et al. Efficacy and safety of dabigatran etexilate and warfarin in "real-world" patients with atrial fibrillation: a prospective nationwide cohort study. I Am Coll Cardiol 2013;61:2264-73. doi:10.1016/j.jacc.2013.03.020

6 Avgil-Tsadok M, Jackevicius CA, Essebag V, et al. Dabigatran use in elderly patients with atrial fibrillation. Thromb Haemost 2016:115:152-60, doi:10.1160/TH15-03-0247.

7 Gorst-Rasmussen A, Lip GYH, Bjerregaard Larsen T. Rivaroxaban versus warfarin and dabigatran in atrial fibrillation: comparative effectiveness and safety in Danish routine care. Pharmacoepidemiol Drug Saf2016;25:1236-44. doi:10.1002/pds.4034.

8 Heidbuchel H, Verhamme P, Alings M, et al. Updated European Heart Rhythm Association Practical Guide on the use of non-vitamin K antagonist anticoagulants in patients with non-valvular atrial fibrillation. Europace 2015;17:1467-507. doi:10.1093/europace/euv309.

9 Olesen JB, Lip GYH, Kamper A-L, et al. Stroke and bleeding in atrial fibrillation with chronic kidney disease. N Engl J Med 2012;367:62535. doi:10.1056/NEJMoa1105594.

10 Kirchhof P, Benussi S, Kotecha D, et al. 2016 ESC Guidelines for the management of atrial fibrillation developed in collaboration with EACTS. Eur Heart/ 2016;37:2893-962. doi:10.1093/eurheartj/ehw210.

11 Nielsen PB, Lane DA, Rasmussen LH, Lip GYH, Larsen TB. Renal function and non-vitamin $\mathrm{K}$ oral anticoagulants in comparison with warfarin on safety and efficacy outcomes in atrial fibrillation patients: a systemic review and meta-regression analysis. Clin Res Cardiol 2015;104:418-29. doi:10.1007/s00392-014-0797-9.

12 Hart RG, Pearce LA, Aguilar MI. Meta-analysis: antithrombotic therapy to prevent stroke in patients who have nonvalvular atrial fibrillation. Ann Intern Med 2007;146:857-67. doi:10.7326/0003-4819-146-12-200706190-00007.

13 Ruff CT, Giugliano RP, Braunwald E, et al. Comparison of the efficacy and safety of new oral anticoagulants with warfarin in patients with atrial fibrillation: a meta-analysis of randomised trials. Lancet 2014;383:955-62. doi:10.1016/S0140-6736(13)62343-0.

14 Schmidt M, Schmidt SAJ, Sandegaard JL, Ehrenstein V, Pedersen L, Sørensen HT. The Danish National Patient Registry: a review of content, data quality, and research potential. Clin Epidemiol 2015:7:449-90. doi:10.2147/CLEP.S91125.

15 Krarup L-H, Boysen G, Janjua H, Prescott E, Truelsen T. Validity of stroke diagnoses in a National Register of Patients. Neuroepidemiology 2007;28:150-4. doi:10.1159/000102143.

16 Gooley TA, Leisenring W, Crowley J, Storer BE. Estimation of failure probabilities in the presence of competing risks: new representations of old estimators. Stat Med 1999;18:695-706. doi:10.1002/ (SICI)1097-0258(19990330)18:6<695\%:AID-SIM60>3.0.CO·2-0.

17 Pisters R, Lane DA, Nieuwlaat R, de Vos CB, Crijns HJGM, Lip GYH. A novel user-friendly score (HAS-BLED) to assess 1-year risk of major bleeding in patients with atrial fibrillation: the Euro Heart Survey. Chest 2010:138:1093-100 doi:10.1378/chest.10-0134.

18 Diener H-C, Aisenberg J, Ansell J, et al. Choosing a particular oral anticoagulant and dose for stroke prevention in individual patients with non-valvular atrial fibrillation: part 2. Eur Heart J 2016ehw069. doi:10.1093/eurhearti/ehw069.

19 Robins JM, Hernán MA, Brumback B. Marginal structural models and causal inference in epidemiology. Epidemiology 2000;11:550-60. doi:10.1097/00001648-200009000-00011.

20 Stürmer T, Wyss R, Glynn RJ, Brookhart MA. Propensity scores for confounder adjustment when assessing the effects of medical interventions using nonexperimental study designs. J Intern Med 2014:275:570-80. doi:10.1111/joim.12197.

21 McCaffrey DF, Griffin BA, Almirall D, Slaughter ME, Ramchand R, Burgette LF. A tutorial on propensity score estimation for multiple treatments using generalized boosted models. Stat Med 2013;32:3388-414. doi:10.1002/sim.5753.

22 Austin PC, Stuart EA. Moving towards best practice when using inverse probability of treatment weighting (IPTW) using the propensity score to estimate causal treatment effects in observational studies. StatMed 2015;34:3661-79. doi:10.1002/sim.6607.
23 Austin PC. Some methods of propensity-score matching had superior performance to others: results of an empirical investigation and Monte Carlo simulations. Biom / 2009;51:171-84. doi:10.1002/ bimj.200810488.

24 Stürmer T, Rothman KJ, Glynn RJ. Insights into different results from different causal contrasts in the presence of effect-measure modification. Pharmacoepidemiol Drug Saf 2006;15:698-709. doi:10.1002/pds.1231

25 Glassock RJ, Rule AD. The implications of anatomical and functional changes of the aging kidney: with an emphasis on the glomeruli. Kidney Int 2012;82:270-7. doi:10.1038/ki.2012.65.

26 Schaeffner ES, Ebert N, Delanaye P, et al. Two novel equations to estimate kidney function in persons aged 70 years or older. Ann Intern Med 2012;157:471-81. doi:10.7326/0003-4819-157-7-201210020-00003

27 Granger CB, Alexander JH, McMurray JJV, et al. ARISTOTLE Committees and Investigators. Apixaban versus warfarin in patients with atrial fibrillation. N Engl J Med 2011;365:981-92. doi:10.1056/ NEJMoa1107039.

28 Halvorsen S, Atar D, Yang H, et al. Efficacy and safety of apixaban compared with warfarin according to age for stroke prevention in atrial fibrillation: observations from the ARISTOTLE trial. Eur Heart) 2014:35:1864-72. doi:10.1093/eurhearti/ehu046.

29 Frost C, Nepal S, Wang J, et al. Safety, pharmacokinetics and pharmacodynamics of multiple oral doses of apixaban, a factor Xa inhibitor, in healthy subjects. Br J Clin Pharmacol 2013;76:776-86. doi:10.1111/bcp.12106.

30 Connolly SJ, Ezekowitz MD, Yusuf S, et al. RE-LY Steering Committee and Investigators. Dabigatran versus warfarin in patients with atrial fibrillation. N Engl J Med 2009;361:1139-51. doi:10.1056/ NEJMoa0905561.

31 Hijazi Z, Hohnloser SH, Oldgren J, et al. Efficacy and safety of dabigatran compared with warfarin in relation to baseline renal function in patients with atrial fibrillation: a RE-LY (Randomized Evaluation of Long-term Anticoagulation Therapy) trial analysis. Circulation 2014;129:961-70. doi:10.1161/ CIRCULATIONAHA.113.003628.

32 Patel MR, Mahaffey KW, Garg J, et al. ROCKET AF Investigators. Rivaroxaban versus warfarin in nonvalvular atrial fibrillation. N EnglJ Med 2011;365:883-91. doi:10.1056/NEJMoa1009638.

33 Harel Z, Sholzberg M, Shah PS, et al. Comparisons between novel oral anticoagulants and vitamin K antagonists in patients with CKD. J Am Soc Nephrol 2014;25:431-42. doi:10.1681/ASN.2013040361.

34 Vestergaard AS, Skiøth F, Lip GYH, Larsen TB. Effect of Anticoagulation on Hospitalization Costs After Intracranial Hemorrhage in Atrial Fibrillation: A Registry Study. Stroke 2016;47:979-85. doi:10.1161/ STROKEAHA.115.012338

35 LaHaye S, Regpala S, Lacombe S, et al. Evaluation of patients' attitudes towards stroke prevention and bleeding risk in atrial fibrillation. Thromb Haemost 2014;111:465-73. doi:10.1160/TH13-05-0424.

36 Lane DA, Lip GYH. Patient's values and preferences for stroke prevention in atrial fibrillation: balancing stroke and bleeding risk with oral anticoagulation. Thromb Haemost 2014;111:381-3. doi:10.1160/ TH14-01-0063.

37 Lip GYH, Lane DA. Stroke prevention in atrial fibrillation: a systematic review. JAMA 2015;313:1950-62. doi:10.1001/jama.2015.4369.

38 Petersen ML, Porter KE, Gruber S, Wang Y, van der Laan MJ. Diagnosing and responding to violations in the positivity assumption. Stat Methods Med Res 2012;21:31-54. doi:10.1177/0962280210386207.

39 Lip GYH, Clemens A, Noack H, Ferreira J, Connolly SJ, Yusuf S. Patient outcomes using the European label for dabigatran. A post-hoc analysis from the RE-LY database. Thromb Haemost 2014;111:933-42. doi:10.1160/TH13-09-0734

40 Hijazi Z, Hohnloser SH, Andersson U et al. Efficacy and Safety of Apixaban Compared With Warfarin in Patients With Atrial Fibrillation in Relation to Renal Function Over Time: Insights From the ARISTOTLE Randomized Clinical Trial. JAMA Cardiol 2016;1:451-60. doi:10.1001/ jamacardio.2016.1170.

41 Grams ME, Plantinga LC, Hedgeman E, et al. CDC CKD Surveillance Team. Validation of CKD and related conditions in existing data sets: A systematic review. Am J Kidney Dis 2011;57:44-54. doi:10.1053/j. ajkd.2010.05.013.

42 Schneeweiss S, Gagne JJ, Glynn RJ, Ruhl M, Rassen JA. Assessing the comparative effectiveness of newly marketed medications: methodological challenges and implications for drug development. Clin Pharmacol Ther 2011;90:777-90. doi:10.1038/clpt.2011.235.

Appendix 1: Supplementary tables A and B Appendix 2: Supplementaryinformation on quality of propensity matching

Appendix 3: Supplementary figures A-E 